\title{
Lithography Process Simulations using OCTA -Application to Development and DSA
}

\author{
Hiroshi Morita \\ Nanosystem Research Inst., National Institute of Advanced Industrial Science and Technology (AIST), \\ Central2-1, 1-1-1 Umezono, Tsukuba, Ibaraki, 305-8568, Japan \\ E-mail:h.morita@aist.go.jp
}

\begin{abstract}
In 2002, the simulation software system for high functional polymeric material OCTA is released. OCTA is the general purpose simulator and each multi-scale-level simulation can be easily done. Recently, we have applied our OCTA system to the problems of lithography. In this paper we discuss the applicability of OCTA system to the simulations for lithography. Two kinds of applicability are discussed. One is the development and the rinse process simulations, and another is the directed self assembly simulation. In the former study, LER can be discussed using OCTA simulation. In the latter study, many kinds of coarse-grained models can be applicable for the DSA simulation using OCTA. These wide applicability are discussed in this paper.
\end{abstract}

Keywords: OCTA, Dissipative particle dynamics, density biased Monte Carlo

\section{Introduction}

In 2002, the simulation software system for high functional polymeric material OCTA [1] is released. After OCTA is released, in Japan, the simulation studies for soft materials are much reported and OCTA is also distributed. Simulation software OCTA played an important role in the community of polymer science. Using OCTA system, we can simulate the materials and the phenomena in the meso scale, which scale is almost from $10 \mathrm{~nm}$ to a few $\mu \mathrm{m}$ scales.

On the other hand, the size of the patterning of the semiconductor becomes smaller and smaller and its size becomes about $10-20 \mathrm{~nm}$. The patterning size used in the industry is now $22 \mathrm{~nm}$ and that in the laboratory is 7-12 $\mathrm{nm}$ using multiple patterning or directed self assembly methods. These sizes become much closer to the size of the polymers, and it becomes much difficult to develop the resist materials. Due to this reason, the simulation is expected as the important tools to develop these materials.

As indicated above, the lithography patterning size is about $10-20 \mathrm{~nm}$ and the most favorable size of our simulation is almost same. We have applied our simulation system to the lithography process since 2008. First we applied the developing step in the lithography process and next applied the rinse step. [2-5] Recently we have applied our system to the directed self assembly (DSA) method. [6,7]
In this paper, the OCTA system is introduced and its potential and applicability to the lithography process simulation is explained. First we explain the application to the development and the rinse processes, and as a second topic, the application study to the directed self assembly process is explained.

\section{OCTA system}

OCTA was developed by Masao Doi and co-workers in 2002, and it is still updating. Latest version is OCTA2013SE, which is released in March, 2014. OCTA contains several simulators and the organized platform. Figure 1 shows the images of OCTA system. The reason why there are many simulators in OCTA is that there are so many coarse-grained models. One of the features of OCTA system is that OCTA is the multi-scale simulation system, and data can be transferred from output file of one simulator to input of another simulator. This facility is one of the important points of OCTA system.

In the lithography process simulations, coarse-grained molecular dynamics simulator "COGNAC" and mean field self consistent field simulator "SUSHI" are used. Using COGNAC simulator, several particle dynamics simulation can be done, such as Kremer-Grest model, or dissipative particle dynamics (DPD) simulations [8] etc., and using SUSHI simulator, self consistent field (SCF) 
[9-14] simulations can be done. In the next section, detail of DPD method is explained.

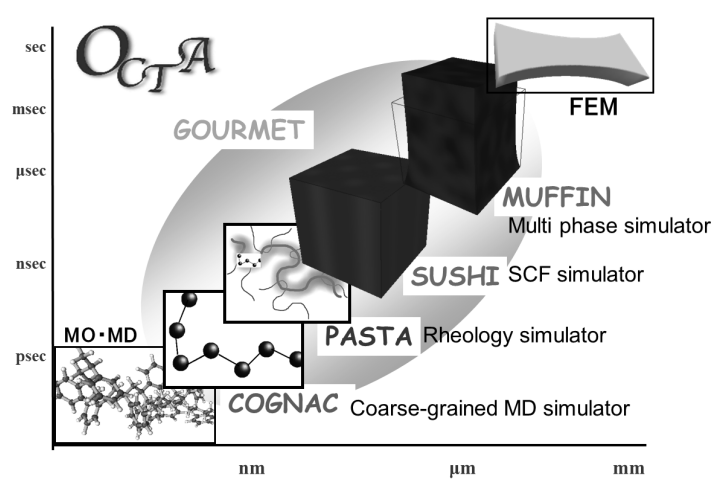

Fig. 1. Overview of OCTA system

\section{Dissipative particle dynamics method}

In the lithography simulations, DPD method is applied. DPD method proposed by Groot and Warren [8] in 1997. DPD method is the particle dynamics simulation and each particles has mass $\mathrm{m}_{\mathrm{i}}$. Equation of motion is written in eq. 1 or 2 ,

$$
\begin{aligned}
& \frac{d \mathbf{r}_{i}}{d t}=\mathbf{v}_{i} \\
& m_{i} \frac{d \mathbf{v}_{i}}{d t}=\mathbf{f}_{i}
\end{aligned}
$$

where $r_{i}$ is the position, and $v_{i}$ is the velocity of the particle $i$.

Force $\mathbf{f}_{\mathrm{i}}$.is described by three kinds of forces; $\mathbf{F}_{\mathrm{ij}}{ }^{\mathrm{C}}$, $\mathbf{F}_{\mathrm{ij}}{ }^{\mathrm{D}}$, and $\mathbf{F}_{\mathrm{ij}}{ }^{\mathrm{R}}$ called as the conservative force, dissipative force, and drag force, respectively.

$$
\mathbf{f}_{i}=\sum_{j \neq i}\left(\mathbf{F}_{i j}^{C}+\mathbf{F}_{i j}^{D}+\mathbf{F}_{i j}^{R}\right) .
$$

$\mathbf{F}_{\mathrm{ij}}{ }^{\mathrm{C}}$ stands for the force arising from the interaction potential. There are two types of interaction potential, the bonding potential and the non-bonding potential as written below;

$$
\begin{gathered}
\mathbf{F}_{i j}^{C}=\left[f^{\text {bond }}\left(r_{i j}\right)+f^{\text {non-bond }}\left(r_{i j}\right)\right] \frac{\mathbf{r}_{i j}}{r_{i j}} \\
f^{\text {bond }}\left(r_{i j}\right)=C r_{i j} \\
f^{\text {non-bond }}\left(r_{i j}\right)=\left\{\begin{array}{cc}
a_{i j}\left(1-\frac{r_{i j}}{r_{c}}\right) & \left(r_{i j}<r_{c}\right) \\
0 & \left(r_{i j} \geq r_{c}\right)
\end{array}\right.
\end{gathered}
$$

where $r_{C}$ is the cut off distance, $a_{i j}$ is a maximum repulsion between particles $i$ and $j$, and $r_{i j}$ is equal to $\left|\mathbf{r}_{\mathrm{i}}-\mathbf{r}_{\mathrm{j}}\right|$. Noted that the $\mathrm{a}_{\mathrm{ij}}$ is related to $\chi$ parameter used in SCF.

The dissipative force and the drag force are given by

$$
\begin{aligned}
& \mathbf{F}_{i j}^{D}=-\gamma \omega^{D}\left(\mathbf{r}_{i j}\right)\left(\mathbf{v}_{i j} \cdot \frac{\mathbf{r}_{i j}}{r_{i j}}\right) \frac{\mathbf{r}_{i j}}{r_{i j}} \\
& \mathbf{F}_{\mathrm{ij}}^{\mathrm{R}}=\sigma \omega^{\mathrm{R}}\left(\mathbf{r}_{\mathrm{ij}}\right) \theta_{\mathrm{ij}} \frac{\mathbf{r}_{\mathrm{ij}}}{\mathrm{r}_{\mathrm{ij}}}
\end{aligned}
$$

where $\theta_{i j}$ is a randomly fluctuating variable with Gaussian statistics, $\omega^{D}, \omega^{R}$, and $\sigma$ are written by

$$
\begin{aligned}
& \omega^{D}\left(r_{i j}\right)=\left[\omega^{R}\left(r_{i j}\right)\right]^{2}=\left\{\begin{array}{cc}
\left(1-\frac{r_{i j}}{r_{c}}\right)^{2} & \left(r_{i j}<r_{c}\right) \\
0 & \left(r_{i j} \geq r_{c}\right)
\end{array}\right. \\
& \sigma^{2}=2 \gamma k_{B} T
\end{aligned}
$$

The most important feature of DPD method is the weak non-bonding interaction, shown in eq. (6). Due to this potential, the polymer chain becomes phantom chain and the simulation becomes accelerated. Therefore we can get the equilibrium structure in shorter simulation time.

In both development and DSA simulations, DPD method is applied. DPD method has several advantages for these simulations compared with other simulation methods. In the development process simulation, the line edge structure with several kinds of roughness such as derived from a single chain conformation can be observed using DPD method. In the DSA simulation, the phase separated structure by high $\chi$ block copolymer can be simulated although it is difficult to be simulated by RPA or SCF methods.

\section{Simulations of Development and Rinse processes}

As a lithography process simulation, the development and the rinse process simulations are picked up. In the development process, the photo resist polymers are dissolved into the developing liquid. In this simulation, the dissolution process simulation has been done by OCTA.

The dissolution process simulation is realized by the change of the phase from two-phase structure (polymer/solvent) to single mixed phase structure. This phase change is controlled by the change of the interaction parameter asp between polymer and solvent. If smaller asp is used, the polymer can be dissolved. The dissolved threshold of asp is about 25.0. Figure 2 and 3 shows the snapshots of the dissolution simulation of the film with asp=15 and 25. In the case of asp=15, the polymer film can dissolve into the solvent phase as shown in Fig. 2. However in the case of asp $=25$, the structure does not changed in Fig. 3. From these simulations, we can be checked that the dissolution can 
be controlled.

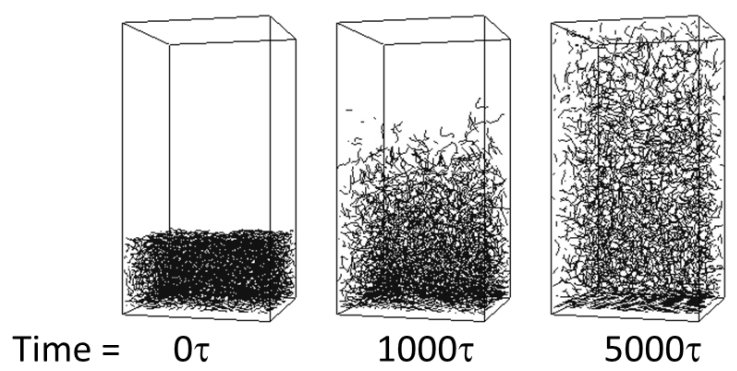

Fig. 2 Snapshots of dissolution simulation with asp $=15$

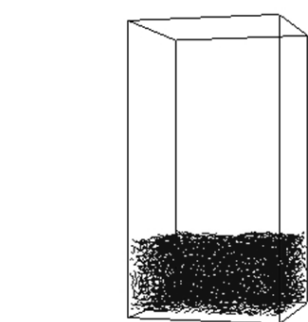

Time $=0 \tau$

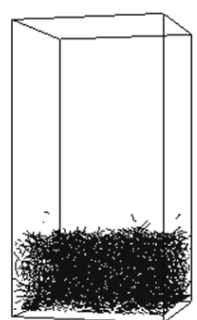

$1000 \tau$

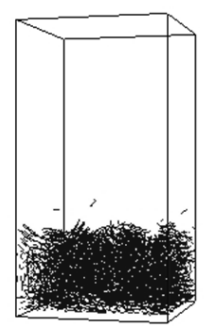

$5000 \tau$
Fig. 3 Snapshots of dissolution simulation with asp $=25$

Next, the developing simulation of the line pattern is performed. In this simulation, the soluble and the insoluble regions are set as the lines. In the real positive tone process, in the photo irradiated area the photo acid generator (PAG) inside the resist film absorbs the photo and the generated acid diffuses in the film. The acid attacks the protected parts of the resist polymer. After this reaction, the polymer becomes soluble. However in our simulation, these processes are skipped and the soluble and the insoluble region are set intensively.

Figure 4(a) shows the initial structure of the developing simulation. The middle region indicates the soluble region. To make the initial structure, the width of the interface is much important. This is because the interfacial width is decided by the length of the diffusion of PAG and its width is much related to the roughness of the line. In our simulation, the soluble and the insoluble region are set by the setting of the interaction parameter asp. Therefore the size of the interfacial width can be set by the change of the interaction parameter asp.

Figure 4 shows the snapshots of the dissolution process simulation. From (a) to (c), the dissolution of the resist polymer proceeds. In this simulation the interfacial width is set as 0 . This means that the patterns can be set as the ideally controlled patterns, which cannot be examined in the real experiments. At the first, the swelling of polymer occurs. The diffusion (a)

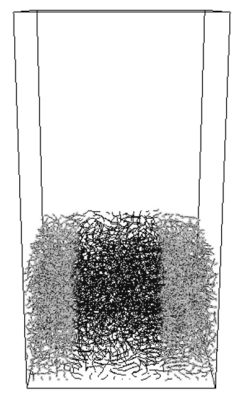

(b)

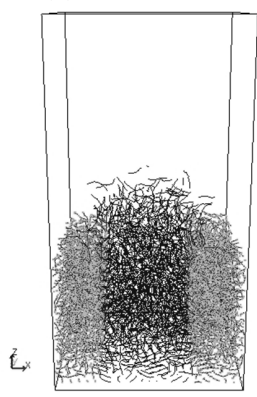

(c)

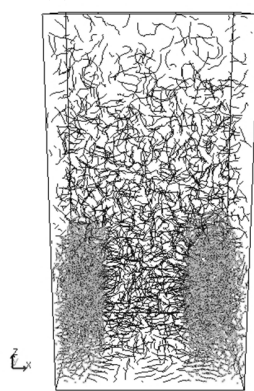

Fig. 4. Simulation results of the dissolution process simulation.

of solvent is much faster than polymer molecules and the penetration of solvent into the film occurs. Next the polymers diffuse into the solvent phase, finally the pattern forms.

In the experiment, Itani and co-workers have performed the fast AFM observation of the dissolution process. [15] From their experiments, the swelling and the diffusion of polymers can also be observed, and our simulation will show one of the projected images of the real processes.

Using our simulations, the LER can also be observed. The roughness derived from the distribution of polymer can be seen. Typical change of the roughness can be found in the rinse process. Next the rinse process simulation is discussed.

Using the structures after the dissolution process simulation, the rinse process simulation is started. In the structure just after the dissolution process simulation, the dissolved polymers are extracted from the system. The spaces of extracted polymers are filled by same number of solvent particles. In this step, the selection of the dissolved polymer molecule is much difficult at the line edge. The polymer chain in which over $30 \%$ of particles are remained in the line is defined as the insoluble polymer chain, and keeps staying in the line.

Figure 5(a) shows the snapshots of the initial structure of the rinse process simulation. Once we obtained the initial structure, we perform the rinse process simulation. The interaction parameter asp is set as 40 . In the rinse process, the polymer does not solve into the rinse liquid and the insoluble value for polymer molecule is used.

As time goes on, the polymers at the line edge becomes shrunk, and the line edge roughness becomes smaller and smaller. Polymers do not favor the rinse liquid, and to reduce the area of the interface, the interface becomes flattened. This result indicates that the LER is recovered in the rinse process, and further 
modification can also be considered to control the rinse process. In the point of view of the dynamics of the polymer chain, the development and the rinse process can be much controlled. (a)

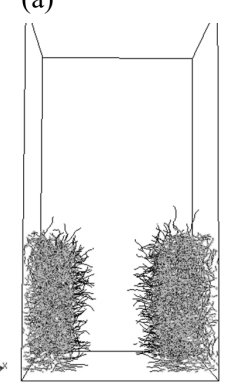

(b)

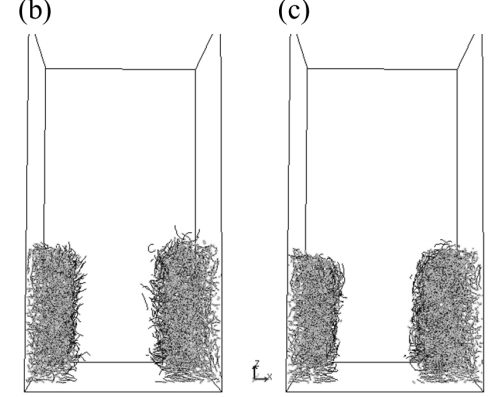

Fig. 5 Snapshots of the rinse process simulation

\section{DSA simulation}

OCTA system can also be applicable to the DSA simulation. There are several kinds of applicable DSA simulations, and some examples are already reported in reference 6 and 7. In OCTA, SCF and DPD simulations can be performed using SUSHI and COGNAC simulators, respectively. And the combinatorial method of both SCF and DPD methods can also be done in OCTA system. This is one of the great advantages of OCTA system. OCTA system is the multi-scale simulation system and the collaboration of several simulators can easily be done. In this paper, the combinatorial method is described.

Using SUSHI simulator, DSA films in two dimensional system on the chemical guide can be simulated as shown in Fig. 6. In the bottom region, chemical guide is introduced. In the upper region, the air particles are also included, and the film is sandwiched between air and chemical guide. In this simulation, $\chi$ parameter between two components of block copolymer is set as 1.0 . The chemical guide is modeled by the "obstacle" option of SUSH simulator. At the surface of "Obstacle," the interaction to the polymer can be added. The attractive layer for the one component of polymer can be realized as the attractive "obstacle" for the one component of polymer and the neutral layer is modeled as the neutral "obstacle" for both components. In this SCF simulation, the density of each segment in a polymer is also calculated. This segment density is used in the construction of initial structure of DPD simulation.

Once the sectional-2D-SCF was done, the initial structure for DPD simulation is constructed using density biased Monte Carlo (DBMC) method. Aoyagi et al [16] developed DBMC method in which the polymer particles set along the statistical weight of previous segment density obtained by SCF simulation, and as the result of Monte Carlo simulation, the initial structure is made. The obtained structure after DBMC method is relaxed by the relaxation DPD simulation and the targeted structure can be simulated. The final structure is shown in Fig. 7. Same structure by SCF simulation can be found.

There are two advantages of this simulation process. One is the fast simulation to obtain the DSA simulation result by DPD. If DPD simulation is started from the random configuration, it takes much more time. In the case of the structure shown in Fig. 7, it takes $45 \mathrm{~min}$. However only using DPD method, it takes $150 \mathrm{~min}$ which is the fastest case. Second advantage is that this method can reduce the risk to simulate the local minimum structure. If you only performed DPD simulation, it is sometimes trapped to the local minimum structure, and it cannot be recovered from the local minimum structure with using the long time relaxation simulation. This method can be free from the simulation of local minimum structure.

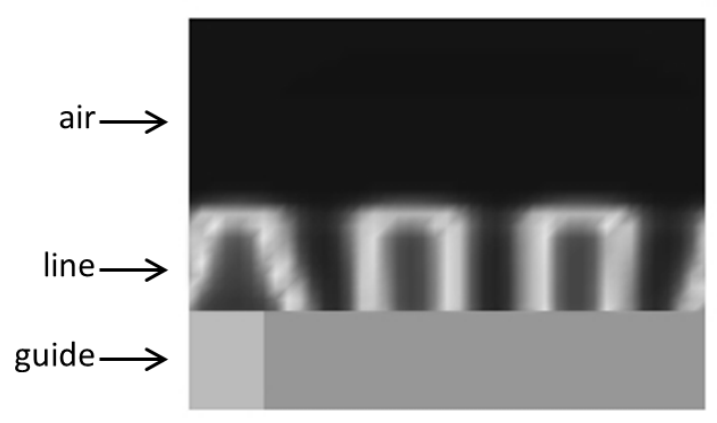

Fig. 6 Sectional 2D simulation using SCF method

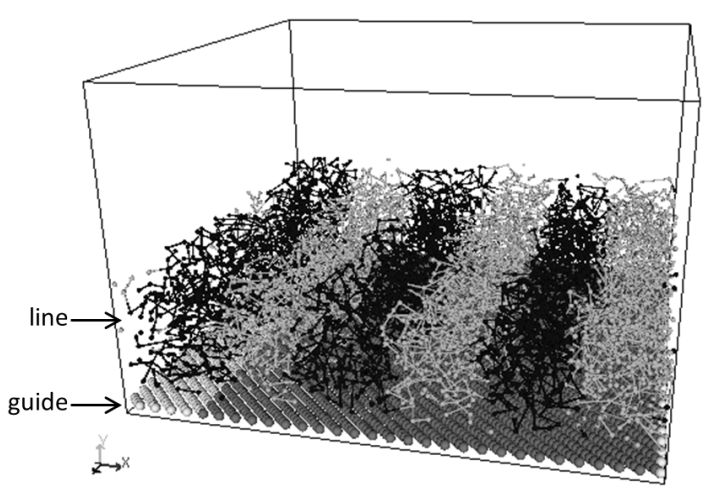

Fig.7 DPD simulation result using chemical guide by SUSHI-DBMC-COGNAC simulations.

DBMC method is only included in OCTA system and this is one of the important advantage using OCTA system. 


\section{Concluding Remarks}

In this paper, the simulations related to the lithography process using OCTA is described. Using OCTA system, the development and the rinse process simulation can be done. From the series of simulations, the dynamics of polymer chain can be simulated and the origin of the roughness by polymer chain can be found from these simulations. We also found the reduction of LER in the rinse process and the further reduction of LER can also be considered.

DSA simulation can also be done by OCTA system. Though it is not written in this paper, the simulations of sectional 2D SCF simulation with physical guide, and topview2D simulation with physical guide can also be done by OCTA system. The applicability of many kinds of models and simulations for DSA is one of the most important feature of OCTA system. The latest version of OCTA is OCTA2013SE, and within 2014, further new version of OCTA will be released. Recently COGNAC and SUSHI simulators can be adapted to the parallel calculation and SUSHI simulator can also be worked on GPU computers. By the evolution of OCTA, we can get more fruitful results by OCTA simulations. We will apply our OCTA system to many kinds of lithography process simulations and will further upgrade our OCTA system continuously.

\section{Acknowledgement}

This study is partially supported by the New Energy and Industrial Technology Development Organization
(NEDO).

\section{References}

1. M. Doi et al., http://octa.jp

2. H. Morita, M. Doi, Proc. SPIE, 7273 (2009) 727337.

3. H. Morita, M. Doi, Proc. SPIE, 7639 (2010)763932.

4. H. Morita, Proc. SPIE, 7972 (2011)79720W.

5. H. Morita, I. Okabe, S. Agarwal, V. K. Singh, Proc. SPIE, 8325 (2012) 83250J.

6. H. Morita, J. Photopolymer Sci. Tech.,26 (2013) 801.

7. H. Morita, Proc. SPIE, 9049, (2014) 9049-97.

8. R. D. Groot, P. B. Warren, J. Chem. Phys., 107 (1997) 4423.

9. M. W. Matsen, F. S. Bates, Macromolecules 29, (1996) 1091.

10. F. S. Bates, G. H. Fredrickson, Physics Today, 52 (1999) 32

11. L. Leibler, Macromolecules, 13, (1980) 1602.

12. M. W. Matsen, M. Schick, Phys. Rev. Lett., 72, (1994) 2660

13. E. Helfand, Z. R. Wasserman, Macromolecules, 9 , (1976) 879.

14. G. J. Fleer, M.A. Cohen Stuart, J.M.H.M. Scheutjens, T. Cosgrove, B. Vincent, "Polymers at Interfaces", Chapman \& Hall, London, (1993).

15. T. Itani, J. J. Santillan, Appl. Phys. Express, 3, (2010) 061601.

16. T. Aoyagi, T. Honda, M. Doi, J. Chem. Phys., 117, (2002) 8153. 